\title{
La gestion en commun des ressources naturelles : une perspective critique
}

Jérôme Ballet

\section{OpenEdition}

Journals

Édition électronique

URL : http://journals.openedition.org/developpementdurable/3961

DOI : 10.4000/developpementdurable.3961

ISSN : 1772-9971

\section{Éditeur}

Association DD\&T

\section{Référence électronique}

Jérôme Ballet, "La gestion en commun des ressources naturelles : une perspective critique ",

Développement durable et territoires [En ligne], Varia (2004-2010), mis en ligne le 29 août 2007, consulté le 19 avril 2019. URL : http://journals.openedition.org/developpementdurable/3961 ; DOI : 10.4000/ developpementdurable.3961

Ce document a été généré automatiquement le 19 avril 2019

\section{(cc) (i) \&)}

Développement Durable et Territoires est mis à disposition selon les termes de la licence Creative Commons Attribution - Pas d'Utilisation Commerciale 4.0 International. 


\title{
La gestion en commun des ressources naturelles: une perspective critique
}

\author{
Jérôme Ballet
}

1 Les politiques de gestion des ressources naturelles, quelles qu'elles soient, visent à maintenir une certaine durabilité des ressources en évitant leur surexploitation, ce qui est traditionnellement appelé la "tragédie des communaux ». Dans une telle optique, la gestion communautaire des ressources naturelles s'est développée comme une voie nouvelle entre la gestion par le marché ou les droits de propriété privée et la gestion étatique. La gestion étatique est souvent fustigée pour sa lourdeur, son manque d'efficacité, mais aussi et surtout pour son incapacité à déterminer et à contrôler les pratiques réelles des populations en matière environnementale. Dans bien des cas, pour les forêts classées dont l'accès est interdit, cette interdiction d'accès sans les moyens de la faire respecter revient à laisser la ressource en accès libre. L'Etat n'étant pas en mesure de faire respecter cette interdiction, les comportements de transgression sont nombreux et produisent une destruction des espaces naturels. De son côté, la gestion par la propriété privée est souvent accusée de créer des inégalités, de produire de l'exclusion, sans pour autant résoudre parfaitement le problème d'épuisement des ressources. Dans un cas comme dans l'autre, les maux sont considérés par les partisans adverses comme nombreux et l'efficacité suffisamment faible.

Dans la recherche de solutions efficaces, le second « Sommet de la terre » qui se déroula à Rio en 1992 a fait ressortir une idée forte : celle de participation de l'ensemble des parties prenantes impliquées dans la mise en œuvre d'une politique. Il s'agissait de dépasser le cadre restreint des décideurs, essentiellement les gouvernements et les industriels via le lobbying, pour inclure la société civile au sens large, c'est-à-dire les associations, les ONG mais aussi plus directement encore les populations locales. De manière pratique, cela se concrétise par des conditionnalités émises sur les projets telles que les populations locales soient impliquées et bénéficient de ces projets, par exemple avec des conditionnalités sur l'embauche prioritaire des populations locales, la création d'infrastructures qui leurs sont bénéfiques (école, coopérative agricole ou artisanale, 
etc.). Plus généralement, ce sommet a mis en avant la notion de "gouvernance participative » qui retranscrit la volonté de faire participer les populations locales à la mise en œuvre de la politique. La gestion en commun des ressources naturelles, en modifiant le régime de propriété, fondant une propriété commune avec ses règles et sanctions, apporte une pièce nouvelle à l'édifice des modes de gestion des ressources, compatible avec l'idée de participation des populations locales.

3 Les institutions internationales se sont emparées de cette question et sont venues à leur tour appuyer cette idée de gouvernance participative. Sur le site de la Banque Mondiale, on peut lire une déclaration de Maurice Strong, secrétaire général de la conférence des Nations-Unies sur l'environnement et le développement en 1992, soulignant avec force que la transition impérative vers le développement durable ne peut se faire qu'avec le support complet des communautés et la participation des personnes ordinaires au niveau local. En 1999, la Banque Mondiale organisait un forum consacré à la soutenabilité environnementale et sociale du développement, combinant ainsi les deux dimensions.

4 Les ONG, fortes de cet ancrage institutionnel et conceptuel, valorisent elles aussi la participation des populations locales. L'organisation de certification des forêts Forest Stewardship Council annonce par exemple que son objectif est de promouvoir une gestion des forêts responsable au point de vue environnemental, socialement bénéfique et économiquement viable. Sur les 10 principes de certification mis en avant, plusieurs points concernent les communautés locales.

5 La gouvernance participative apparait comme un projet politique relayé aux différents niveaux des instances de décisions. Soulignant le rôle essentiel des populations locales dans la gestion des politiques, le concept appuie l'idée d'une gestion en commun des ressources naturelles. Ce mode de gestion est supposé efficace. La gestion communautaire des ressources naturelles s'appuie sur un ancrage territorial visant à donner sens à la notion de communauté. Elle n'évite cependant pas un nombre de risques non négligeable relatifs à la manipulation, au jeu de pouvoir et aux comportements opportunistes. Certes, ces jeux d'acteurs ne sont pas l'apanage des communautés locales ou des sociétés traditionnelles et se retrouvent dans toutes les communautés, groupes de travail, entreprises, etc., mais on ne peut pas faire comme si les communautés locales faisaient exception à la règle.

6 Il ne s'agit pas dans cet article de remettre en cause la pertinence de cette approche, mais de mettre en garde contre certaines utilisations abusives ne prenant pas les précautions nécessaires à sa mise en application ${ }^{1}$.

7 La discussion critique de certaines utilisations s'appuiera plus particulièrement sur deux aspects. Premièrement, la gestion communautaire des ressources naturelles se réfère à une communauté au sens d'un ensemble de populations localement identifiées par un espace géographique. Or, cette identification pose de sérieuses difficultés puisqu'elle n'implique pas que la communauté soit homogène, et laisse la place à des stratégies individuelles de pouvoir et d'exclusion dont les effets sur l'environnement peuvent être néfastes. Il en découle alors une efficacité très discutable puisque les comportements de passagers clandestins ne seront probablement pas éliminés, mais surtout les comportements opportunistes visant à l'utilisation des ressources à des fins personnelles ne sont pas exclus. Dans une première section, un bref rappel de la théorie et de la mise en application de la gestion en commun des ressources naturelles est proposé. Dans une seconde section, la question de l'homogénéité ou de l'hétérogénéité de la communauté et de l'impact que cela exerce sur la gestion des ressources est discutée. Dans une troisième 
section, les risques de comportements déviants sont analysés. Enfin, une quatrième section conclut sur le risque d'instrumentalisation de la démarche.

8 La gestion en commun des ressources naturelles se situe dans la perspective du principe de subsidiarité. Selon Mors (1993), ce principe consiste à choisir un niveau hiérarchique d'exécution des politiques minimisant les coûts économiques et maximisant le bien-être social. Il repose donc sur l'idée d'efficacité dans l'exécution des politiques. Le principe de subsidiarité appelle donc à une délégation effective de pouvoir au niveau le plus bas. Dans cette optique, la société civile peut être mobilisée pour l'accomplissement d'actions qui visent l'intérêt général; les communautés étant une partie de la société civile. La communauté locale apparaît en fait comme le niveau approprié pour la gestion des ressources. Il faut entendre par communauté locale un ensemble de personnes territorialement localisées. Autrement dit, la communauté se définit dans cette optique par un ancrage territorial. Cependant, cet ancrage territorial est aussi censé donner sens aux valeurs partagées par cette même communauté. Cet amalgame constitue un problème central. Nous développerons ce point de vue dans les sections suivantes.

9 La délégation de pouvoir au niveau le plus bas s'appuie sur l'idée d'efficacité de ce niveau hiérarchique pour la gestion des ressources. Elle est justifiée par deux arguments complémentaires : la possibilité de définir des droits de propriété commune et la capacité de la communauté locale à faire respecter ces droits et éviter les comportements déviants.

Avant de devenir un axe important des programmes de gestion des ressources naturelles, la gestion en commun a surtout connu une vive remise en cause avec l'article de Hardin (1968) sur la "tragédie des communaux ». Cet article s'appuie sur l'hypothèse centrale selon laquelle l'absence de droits de propriété provoque la surexploitation des ressources. Définir des droits de propriété devient alors l'enjeu essentiel afin de réguler l'utilisation des ressources. Or, dans ce domaine, la propriété privée apparaît comme la solution la plus simple puisque, comme le soulignent les partisans de l'école des droits de propriété, l'aspect majeur des ces droits et leur fonction première consistent à créer une incitation forte à l'internalisation des externalités (Demsetz 1967).

11 Cependant, la capacité des droits de propriété privée à réguler l'utilisation des ressources peut être mise en défaut si la capacité de contrôle de ces droits est réduite. Il faut en fait distinguer entre les droits de jure ou droits nominaux et les droits de facto ou droits effectifs ou réels. L'insécurité qui pèse sur les droits de jure, en raison du coût prohibitif nécessaire pour établir leur respect, constitue une source importante d'inefficience vis-àvis de l'objectif de régulation de l'utilisation des ressources. Si ce problème est non négligeable dans les pays développés en raison des coûts de transaction qui affectent la capacité de contrôle, il devient majeur dans les pays en développement dès lors que s'ajoutent aux coûts de transaction des phénomènes d'accaparement arbitraire des droits, une insuffisance des infrastructures, et des conflits entre les droits légaux et les coutumes (Platteau 1996). Dans de nombreux cas, l'internalisation des externalités par les droits de propriété privée sera de fait réduite et n'évitera pas la surexploitation des ressources.

De manière très classique, pour résoudre les défaillances des droits de propriété et du marché, le recours à l'intervention de l'Etat a souvent été perçu comme la solution idéale. Les droits de propriété sont alors transférés à l'Etat qui devient gardien des ressources. Cependant, dans de nombreux cas, tels les forêts classées, la capacité de l'Etat à assurer un contrôle de fait sur l'accès aux ressources est très limitée. Le transfert de la propriété de jure à l'Etat ne garantit en rien que la propriété de facto soit mieux respectée. Les 
ressources se trouvent finalement sous l'emprise d'une tragédie de l'accès libre (Ault et Rutman 1979, Bassett 1993, Weber 2000).

13 Face aux défaillances de la propriété privée et de l'Etat, la gestion en commun réapparaît comme une solution et ce d'autant plus que son inefficience présumée dans la lignée de Hardin est due à une interprétation abusive de la notion de " commun " s'appuyant sur une confusion entre l'absence de propriété et la propriété collective (Ciriacy-Wantrup et Bishop 1975). La tragédie des communaux s'exerce dans le cas d'absence de droits de propriété mais pas nécessairement dans celui de propriété commune. La propriété commune, contrairement à l'absence de propriété, se réfère à un ensemble de règles définies en lien avec l'appartenance des membres à une communauté. Baland et Platteau (1996) font une distinction entre les propriétés communes régulées et les propriétés communes non régulées. Les propriétés communes non régulées sont protégées seulement par les restrictions portant sur le fait d'être membre ou non de la communauté, sans règle de conservation stricte, tandis que les propriétés communes régulées s'appuient à la fois sur une restriction de l'usage aux seuls membres et des règles d'usage appliquées aux membres.

14 La gestion en commun des ressources naturelles pourra alors être considérée comme efficiente dès lors que la propriété commune garantira mieux que la propriété privée ou l'Etat la propriété de facto. La capacité de contrôle de l'utilisation des ressources par les communautés locales devient l'enjeu essentiel.

15 La propriété commune est-elle en mesure de garantir une utilisation soutenable des ressources? Autrement dit, est-elle en mesure de réduire les comportements opportunistes des individus dans l'accaparement des ressources? Différents arguments sont venus l'appuyer. Tout d'abord, la compilation d'études de terrain a servi de base à la construction d'une démonstration de l'efficacité des communautés rurales, à partir de normes traditionnelles, dans la gestion de leurs ressources et ce en dehors de toute intervention de l'Etat (Ostrom 1990). De là Pretty et Ward (2001) n'hésiteront pas à franchir un pas de plus en formulant la proposition inverse selon laquelle la disparition des institutions locales a pu entraîner la dégradation des ressources naturelles.

16 Ensuite, les négociations menées entre plusieurs partenaires dans le cadre de la gestion des ressources naturelles montrent généralement, en raison des intérêts divergents de ces partenaires, des comportements de passagers clandestins et l'aboutissement à un consensus instable peu favorable à une bonne gestion (Ostrom et al. 1994). D'où l'idée qu'il faut trouver une instance de négociation et de décision au niveau de laquelle le désengagement conduit à des coûts de rupture ou de déviance significatifs. Il faut donc chercher une instance qui permette une communauté d'actions dans un contexte identifié et pour un projet limité (Ollagnon 1991). Les communautés locales apparaissent alors comme l'instance adéquate puisque les normes communautaires produisent généralement des coûts significatifs pour les personnes ne les respectant pas (exclusion de la communauté par exemple).

17 Enfin et surtout, c'est l'argument du capital social au sein des communautés qui appuie le plus largement le recours à cette instance de gestion. Le capital social est entendu ici comme un ensemble de valeurs communes. La présence d'un fort capital social est une des conditions retenues par la plupart des théoriciens de la gestion en commun des ressources naturelles (Wade 1988, Ostrom 1990, Baland et Platteau 1996, Isham 2000, Pretty et Ward 2001, Platteau 2003 pour ne citer que quelques auteurs). Le capital social aurait pour effet d'accroître fortement l'efficacité des démarches participatives et 
rendrait l'action collective possible en éliminant les comportements déviants et de passagers clandestins, en réduisant les coûts de transaction. Dans cette optique, un lien direct est établi entre l'existence d'un fort capital social et la confiance mutuelle des acteurs. La confiance mutuelle incite chacun des acteurs à se comporter conformément aux normes puisque chacun anticipe que les autres s'y conformeront. Chaque usager agit dans le respect du maintien de la ressource naturelle parce qu'il anticipe que les autres feront de même. La confiance permet ainsi de réduire les coûts de gouvernance, c'est-àdire les coûts qui pèsent sur toutes formes de propriétés collectives et qui concernent la mise en place de l'action collective ou la recherche de compromis dans la régulation de l'usage de la ressource (Platteau 2003).

La gestion en commun des ressources naturelles apparaît en conséquence comme une solution efficace à la tragédie des communaux pour deux raisons. D'une part, elle suppose la participation de la population locale, ce qui finalement, par les mécanismes d'implication de cette population, réduit les effets de mauvaises utilisations ou d'utilisation abusive. D'autre part, les valeurs ou normes utilisées au niveau local réduisent les comportements de passagers clandestins du fait des contraintes qui pèsent sur les individus et des risques associés à la déviance vis-à-vis de ces normes.

La gestion en commun des ressources naturelles s'appuie sur la participation des populations locales. Or, au-delà de la volonté politique et des arguments théoriques, il existe en fait une multiplicité de formes de gestion participative se recoupant plus ou moins, donnant un ensemble d'arrangements institutionnels variés. Comme le soulignent Barrett et al. (2001) et Berkes (2006), la gestion en commun des ressources naturelles ne concerne pas seulement les - et ne peut être réduite aux - communautés locales, elle est en lien avec la question de la gouvernance à de multiples niveaux impliquant des interactions entre les différents niveaux. Méral (2004) distingue cinq catégories de gestion participative regroupées dans le tableau n ${ }^{\circ} 1$.

Tableau $n^{\circ} 1$ Catégories de gestion participative

\begin{tabular}{|l|l|l|l|}
\hline $\begin{array}{l}\text { Intitulé } \\
\text { français }\end{array}$ & $\begin{array}{l}\text { Intitulé en } \\
\text { anglais }\end{array}$ & Descriptif \\
\hline \hline Gestion de terroir & $\begin{array}{l}\text { Village Land Use } \\
\text { Planning }\end{array}$ & $\begin{array}{l}\text { La gestion de terroir repose sur l'association des acteurs } \\
\text { locaux à la gestion proprement dite du terroir. Elle } \\
\text { n'implique pas a priori de dispositif institutionnel } \\
\text { particulier. Le concept de gestion de terroir repose } \\
\text { essentiellement sur la mise en place de dispositifs } \\
\text { permettant d'associer les populations locales à la } \\
\text { planification des activités collectives. L'objectif de la } \\
\text { gestion du terroir n'est pas forcément lié à la gestion des } \\
\text { ressources naturelles même si cette dernière occupe une } \\
\text { place importante dans les pays africains. } \\
\text { Aujourd'hui la gestion de terroir est souvent associée à } \\
\text { la notion de développement local et de décentralisation. }\end{array}$ \\
\hline
\end{tabular}




\begin{tabular}{|c|c|c|}
\hline $\begin{array}{l}\text { Gestion } \\
\text { communautaire } \\
\text { des ressources } \\
\text { naturelles }\end{array}$ & $\begin{array}{l}\text { Community } \\
\text { Based } \\
\text { Natural } \\
\text { Resources } \\
\text { Management }\end{array}$ & $\begin{array}{l}\text { Repose sur un transfert des pouvoirs de gestion des } \\
\text { ressources naturelles ou de l'environnement. La gestion } \\
\text { est collective, dirigée par un groupe d'acteurs et } \\
\text { disposant d'un réel pouvoir de décision. La participation } \\
\text { est active. La GELOSE (Gestion Locale Sécurisée) s'inscrit } \\
\text { dans cette catégorie. A noter qu'à la différence de la } \\
\text { cogestion (voir ci-dessous), la foresterie communautaire } \\
\text { appartient à cette catégorie lorsque les rapports avec les } \\
\text { services forestiers ne sont pas essentiels à la gestion de } \\
\text { l'écosystème forestier (cas des droits d'usage par } \\
\text { exemple). }\end{array}$ \\
\hline Cogestion & $\begin{array}{l}\text { Joint Forest } \\
\text { Management }\end{array}$ & $\begin{array}{l}\text { Tout type de gestion où les choix sont faits } \\
\text { conjointement par une catégorie des acteurs locaux et } \\
\text { les services forestiers. La GCF (Gestion Contractualisée } \\
\text { des Forêts) peut être qualifiée de JFM. }\end{array}$ \\
\hline $\begin{array}{l}\text { Gestion } \\
\text { collaborative (ou } \\
\text { conjointe) }\end{array}$ & $\begin{array}{l}\text { Collaborative } \\
\text { management ou } \\
\text { co-management }\end{array}$ & $\begin{array}{l}\text { Tout type de gestion où les choix sont faits par les } \\
\text { services compétents après avoir obtenu l'accord des } \\
\text { acteurs locaux. }\end{array}$ \\
\hline $\begin{array}{l}\text { Gestion } \\
\text { adaptative }\end{array}$ & $\begin{array}{l}\text { Adaptative } \\
\text { management }\end{array}$ & $\begin{array}{l}\text { En règle générale, il s'agit d'une gestion collaborative } \\
\text { dont les décisions sont évaluées en cours d'application et } \\
\text { donne lieu à des réorientations discutées avec les } \\
\text { acteurs locaux. } \\
\text { Parfois, ce type de gestion se distingue de la précédente } \\
\text { par l'introduction des scientifiques dans les processus de } \\
\text { décision. Dans ce cas, l'accent est mis sur (1) } \\
\text { l'incomplétude de l'information qui (2) limite le rôle de } \\
\text { l'expert / scientifique dans les choix à réaliser. Ce } \\
\text { constat permet de mettre en avant (3) l'accroissement } \\
\text { des connaissances qui se fait en continue et qui (4) } \\
\text { implique une réorientation progressive des choix sur } \\
\text { une base participative. }\end{array}$ \\
\hline
\end{tabular}

Source : Méral (2004).

20 Cette pluralité de formes souligne bien que la gestion en commun des ressources naturelles s'insère dans une perspective politique large visant à la décentralisation et à la participation des populations locales aux processus de décision. Cependant, elle souligne aussi que la participation des populations locales est envisagée de manière pratique sous différentes perspectives et avec une implication plus ou moins forte. Dans certains cas, la participation se limite à une simple consultation telle la gestion collaborative, tandis que dans d'autres cas l'implication suppose aussi le pouvoir de décision, ce qui semble être le cas de la gestion communautaire des ressources naturelles.

21 La pluralité peut retranscrire deux interprétations divergentes. Dans le premier cas elle retranscrit le fait que la plupart du temps un mode de gestion spécifique comme la gestion publique, la gestion privée ou la gestion communautaire, n'est pas en mesure de régler l'ensemble des problèmes et que l'articulation des différents modes de gestion 
formant une configuration institutionnelle chaque fois spécifique permet d'appréhender au mieux la spécificité du cas à traiter. Agrawal (2005) a, par exemple, souligné que le gouvernement pouvait jouer un rôle essentiel en matière de régulation de l'utilisation des ressources en impulsant les mécanismes participatifs tels les conseils de forêts. Acciaioli (2006) témoigne d'un cas d'arrangement institutionnel original entre les traditions coutumières, l'intervention moderne des ONG et le cadre institutionnel défini par l'Etat, dans la gestion des parcs nationaux en Indonésie.

Dans le second cas, la pluralité des formes retranscrit le peu de considération qui est accordée à la gestion communautaire qui apparaît comme une tendance obligée par le discours à la mode, mais dont la prise en compte reste fortement conditionnée, non pas pour des raisons d'efficacité mais pour des raisons de légitimité, à la soumission d'une gestion publique.

Dans un cas comme dans l'autre, la participation peut s'avérer relativement limitée. Froger et al. (2004) soulignent parfaitement ce point de vue en distinguant trois formes de gouvernance participative correspondant à trois niveaux d'implication dans la mise en œuvre de la politique : la participation passive, la participation active et la participation responsable.

La participation passive se caractérise uniquement par un processus de consultation des populations sous forme de recueil des opinions sur l'état de la situation et éventuellement les actions à mener. Cette forme de participation ne garantit évidemment absolument pas que les opinions émises seront prises en compte dans la décision finale. De plus, cette forme de participation repose non seulement sur une structure décisionnelle verticale mais aussi sur une structure d'information verticale. Les populations locales sont en effet souvent informées des projets via des relais politico-administratifs et coutumiers. Le projet est généralement présenté aux populations par les élus ou représentants locaux des partis politiques ou encore par les systèmes de chefferies traditionnelles, appuyant le caractère bénéfique des projets et les risques de sanctions pouvant se réaliser en cas de refus de sa mise en œuvre. La consultation se résume parfois, pour ne pas dire souvent, à convaincre les populations du bienfait et de l'intérêt du projet mis en place; ces dernières n'ayant guère intérêt à contester les décisions prises par la hiérarchie socio-politique, sauf à encourir des sanctions futures.

La participation active pousse le degré de participation plus loin. Elle suppose que des représentants des populations participent réellement à l'élaboration et à l'exécution des projets. Cependant, reste ouverte la question de la nature de ces représentants et de leur réelle représentativité. Bien souvent, ils restent issus de la structure socio-politique et ne procèdent pas d'une élection au sein de la population pour désigner les porteurs de projets. En ce sens, si la participation est active dans sa forme elle ne l'est guère sur le fond.

La participation responsable franchit un degré d'implication de plus en s'appuyant sur l'autonomie des acteurs locaux qui vont bénéficier d'un transfert d'autorité dans la gestion des ressources. Les projets sont alors bâtis sur un partenariat entre le gouvernement et les populations locales, ou plus exactement leurs représentants. Ce partenariat repose sur une confiance dans les capacités de gestion de la part des communautés, ce qui a pour effet de libérer l'Etat d'un certain nombre de fonctions et activités et suppose une décentralisation suffisamment avancée. De ce point de vue, cette dernière forme paraît nettement supérieure aux autres. Pourtant, elle reste loin d'un processus démocratique à la base puisque si ce sont des représentants des populations qui 
gèrent le projet, aucune garantie n'est donnée sur le mode d'attribution d'un pouvoir de représentation.

Qu'il s'agisse donc d'une forme ou l'autre la participation des populations locales peut rester assez faible. Il faut en fait entendre, la plupart du temps, par participation de la population locale l'utilisation de mécanismes et de règles au niveau local, relayés par des autorités locales dont la légitimité reste une question totalement ouverte.

La gestion en commun des ressources naturelles procède d'un ancrage territorial fort dans lequel une implication de la population locale, via le concept de gouvernance participative, constitue le point d'appui. Au-delà des limites qui viennent d'être émises sur la réalité de cette participation, une ambiguïté est au cœur de cette démarche. En ciblant une population locale, elle suppose en fait que les institutions locales (au sens des règles, coutumes, habitudes, etc.) sont relativement partagées ou qu'au moins, les populations localement regroupées seront incitées voire contraintes à suivre ces institutions par le pouvoir de sanction supposé par la forte imbrication des populations sur un territoire restreint. De ce fait, lorsqu'une politique passe par le relais des chefferies traditionnelles ou par toute autre institution locale, cela revient à considérer que toute la population suivra les décisions prises et se soumettra à ces institutions. Un tel point de vue permet de comprendre qu'il n'est pas nécessaire que la gouvernance participative corresponde à une véritable gouvernance démocratique à la base. Il suffit d'établir des relais et que ces relais constituent des autorités locales suffisantes pour la gestion des ressources.

29 La communauté locale est donc conçue comme une unité de base. Elle n'a pas besoin d'être homogène dans le sens où tout le monde aurait le même pouvoir, il suffit que les règles établies s'appliquent à tout le monde de manière suffisamment contraignante ${ }^{2}$. C'est finalement ce postulat qui implique l'efficacité de la gestion en commun. Or un tel postulat pose de sérieux problèmes.

Premièrement, la communauté n'est pas une donnée objective dans le sens où l'on pourrait regrouper tous les individus ayant certaines caractéristiques objectivement perceptibles dans un même ensemble. La communauté est avant tout subjective. Selon Mahieu (2001), la communauté de chaque individu, et non à laquelle appartient chaque individu, est un construit en fonction de son âge, de son sexe, de sa situation familiale, de sa religion, de son ethnie, et des relations qu'il développe. Si l'adhésion à une communauté implique un certain nombre de contraintes, elle laisse aussi la place à des stratégies individuelles, et ce d'autant plus que la communauté n'est pas donnée objectivement mais découle aussi d'un construit sur lequel chaque individu a une certaine emprise. Les contraintes qui pèsent sur l'individu résultent de réalités tangibles telles que les engagements économiques que certains prennent en entrant en relation avec d'autres personnes, mais également d'un monde imaginaire et de l'incertitude. «La crainte de l'obligation vient de ce que la communauté appartient non seulement à un monde réel, mais aussi à des mondes imaginaires » (Mahieu 2001, p. 258). Ces mondes imaginaires sont du ressort de la psychologie et/ou de la sorcellerie. Dans bien des cas, ils impliquent une incertitude radicale sur les sanctions qui peuvent tomber sur l'individu qui ne se soumettrait pas aux contraintes communautaires. Ces sanctions peuvent être économiques avec l'absence d'assistance en cas de besoin, psychologiques si les individus craignent de perdre la face, mais aussi physiques avec les maladies imputées à la sorcellerie. Ainsi, selon De Rosny (1981), certaines personnes sont amenées à développer un véritable complexe de persécution. 
31 Cependant, et malgré les sanctions possibles, réelles ou imaginaires, certains individus manipulent l'information, trichent sur leur état, et tentent de développer des stratégies individuelles au sein de leur communauté. De sorte qu'au sein d'une communauté, chaque individu est contraint, mais développe également des stratégies qui lui permettent de tirer profit de sa communauté. Citons sur ce point l'anthropologue Daniel Pelligra (1998, p. 79) : « Devrai-je élucider la part d'initiative personnelle, d'autonomie, de personnalité des individus face à la norme et jusqu'où, avec quels prétextes, avec quelles compensations, on peut s'autoriser à la contourner ».

32 Admettre qu'au sein d'une communauté les individus puissent manipuler l'information, jouer de leur autonomie pour contourner les normes, implique qu'ils développent des stratégies individuelles et ne se comportent pas comme s'ils étaient tous unis dans un même objectif. La communauté n'est pas une unité, elle est composée de multiples individus aux stratégies parfois fort différentes. En ce sens, parler de gestion en commun cache la réalité du problème puisqu'il s'agit plutôt d'une gestion à partir de règles communautaires dont certains membres peuvent bénéficier et d'autres pâtir. La gestion communautaire des ressources naturelles ne peut faire l'économie des stratégies individuelles et de leur impact sur l'efficacité de la gestion.

Deuxièmement, il découle du point précédent que le capital social n'est pas nécessairement une variable unificatrice. Le capital social fait l'objet d'investissements et de stratégies d'investissement ayant pour objectif l'accumulation de pouvoirs et l'acquisition d'autres formes de capital (économique principalement). Portes (1998) exprime un point de vue similaire. Il prend en considération la façon dont un individu profite de ses caractéristiques personnelles pour renforcer son pouvoir et maximiser les ressources tirées du réseau, et la façon dont un individu profite de sa position dans le réseau pour renforcer son pouvoir personnel. Les trois formes de pouvoir systématiquement mobilisées seraient : la coercition, l'autorité et le charisme. De même, l'exemple de Coleman (1998) sur les clubs de sénateurs illustre parfaitement cette idée. Dans les clubs de sénateurs, certains sont plus influents que d'autres parce qu'ils ont su construire un ensemble d'obligations vis-à-vis des autres sénateurs et peuvent en conséquence utiliser les dettes des autres à leur égard pour faire passer leur proposition de loi. Ainsi, le pouvoir ou l'influence exercée sur les autres permet de faire aboutir plus facilement les projets. D'autres études convergent vers ce point de vue. Ballet et Hamzetta (2003) ont par exemple montré, dans le cas de la Mauritanie, en quoi les individus pouvaient tirer profit des réseaux et de leur appartenance communautaire en s'appuyant sur leur statut et reproduire ainsi les inégalités. Dans une telle optique, l'information devient un facteur central du pouvoir. Les trous structuraux (Burt 1992) dans un réseau ou une communauté permettent d'obtenir des avantages sur les autres membres $^{3}$. La gestion participative passant par les règles traditionnelles ou locales, minimisant ainsi la démocratie réelle, renforce ou au contraire perturbe les situations de pouvoir au niveau local et dans tous les cas fait l'objet de stratégies et de processus de manipulation et d'utilisation à des fins personnelles. Ces stratégies personnelles et le statut qu'acquièrent les individus dans la communauté rendent alors l'efficacité de la gestion en commun très relative.

Troisièmement, l'hétérogénéité des communautés renvoie à la possibilité ou non de mener une action collective compatible avec une bonne gestion des ressources. L'hétérogénéité des communautés locales peut être considérée, non pas comme un problème pour la gestion des ressources, mais au contraire comme un atout. Dans la 
lignée de la logique de l'action collective proposée par Olson (1965), l'hétérogénéité d'un groupe peut constituer un avantage pour la réalisation d'une action collective. Un groupe fortement homogène se heurte au problème classique soulevé par Olson selon lequel le coût individuel de l'action implique que chacun attende que les autres agissent puisque personne ne peut être exclu du bénéfice du bien. Dans une communauté hétérogène, les individus les mieux nantis peuvent supporter les coûts de l'action pour une part proportionnellement plus élevée de bénéfice que les moins bien nantis (Baland et Platteau 1999). Jodha (1985) suggère par exemple, dans le cas des zones semi-arides d'Inde durant la période coloniale et pré-coloniale, que le succès de l'action collective visant à préserver les ressources naturelles a été rendu possible par la concentration du pouvoir aux mains d'une classe sociale quasi féodale et par l'exclusion des populations pauvres et marginalisées.

A l'opposé de cette hypothèse, l'hétérogénéité peut être considérée comme un frein essentiel à la bonne gestion des ressources. L'inégalité dans la répartition du pouvoir de décision (Neupane 2003), le faible degré de confiance qu'implique l'hétérogénéité (Seabright 1993) constituent des éléments essentiels de dysfonctionnement dans la gestion des ressources. Dans certains cas l'hétérogénéité réduit toute capacité à s'autoorganiser collectivement puisque les plus nantis satisfont leurs besoins par le biais de ressources privées tandis que les moins nantis ne sont pas en mesure de supporter les coûts de l'action collective (Agrawal 1993, Mukhopadhyay 2004).

Pour sa part, McPeak (2005) argumente que l'hétérogénéité économique parmi les utilisateurs de la ressource ne peut constituer une variable décisive et que l'hétérogénéité spatiale doit être prise en compte sérieusement. Cependant, au-delà des argumentaires sur le niveau souhaitable d'hétérogénéité ou d'homogénéité, il est clair que l'efficacité de la gestion en commun est très dépendante de la structuration communautaire et des stratégies individuelles de pouvoir au sein de la communauté. Fauroux (2003) a montré de manière remarquable dans le cas de Madagascar que le pouvoir local en milieu rural est bâti sur des structures complexes et hétérogènes. Il en résulte plusieurs types de rapports de force dont les répercussions se font ressentir sur la gestion des ressources telles les forêts, les pâturages et l'eau. Pour citer Fauroux dans le cas de l'eau : «La gestion de l'eau d'irrigation suscite d'incessants problèmes et parvient, à elle seule, à empoisonner les relations sociales locales. C'est au point que, souvent, chaque lignage préfere creuser son propre canal, ce qui donne le spectacle étrange de quatre ou cinq petits canaux précaires s'allongeant côte à côte sur plusieurs kilomètres. S'il existe un canal commun, les associations d'usagers de l'eau sont déchirées par d'insolubles conflits»(2003, p. 12). Cet exemple illustre parfaitement les aspects symboliques du pouvoir au sein d'un territoire très restreint et les répercussions que ces aspects peuvent avoir sur les modes de gestion des ressources.

37 Les arguments qui viennent d'être avancés soulignent évidemment que les stratégies personnelles peuvent être des stratégies opportunistes et que dans un tel cadre la gestion des ressources naturelles peut en pâtir. Une telle optique semble bien se vérifier dans un certain nombre de cas. Au-delà des problèmes immédiats de gestion que cela cause sur les ressources, des implications sur la communauté locale elle-même se feront ressentir. Les détenteurs de pouvoir confrontés à une dégradation des ressources pourront adopter une stratégie de maintien au pouvoir en dénonçant des responsables de cette dégradation, même si et surtout si bien sûr ils sont eux-mêmes, en tant que garants de la politique de gestion, les co-responsables de cette mauvaise gestion. 
38 Agrawal (1994) et Andersen (1995) révèlent comment, en Inde, les villageois de castes supérieures s'approprient les ressources communes pour leur bénéfice personnel au détriment des villageois les plus pauvres, conduisant à une perte de confiance au sein de la communauté, avec des effets généralement néfastes sur la gestion de la ressource. Ribot (1995) décrit le même type de conflit dans le cas du Sénégal. Nugent (1993) note pour sa part que la gestion en commun des ressources naturelles donne lieu à des stratégies de recherche de rente au sein des organisations locales provoquant une relative inefficience dans la gestion. Daniere et Takahashi $(1997,1999,2000)$ analysent quant à eux les valeurs en Thaïlande et en déduisent leur efficacité dans la gestion environnementale communautaire des projets. Leur analyse empirique consiste à évaluer l'impact de cinq valeurs, considérées comme clé en Thaillande, sur la gestion environnementale communautaire à Bangkok. La première de ces valeurs est l'évitement (avoidance). Elle consiste pour une personne à éviter les conflits et les controverses. Cette valeur pourrait jouer un rôle négatif dans la mesure où elle implique un conformisme des individus qui pourraient dans ce cas nier l'existence de problèmes si ceux-ci nécessitent une réaction et prise de position. Ils appuient cette possibilité avec le cas de la contamination de l'eau des canaux de Bangkok. La seconde valeur est l'individualisme. L'individualisme signifie ici, pour une personne, l'absence d'obligations à l'égard des institutions sociales et des autres individus. La troisième valeur est la relation patron-client. Il s'agit d'une valeur de réciprocité. Le patron assiste son client en échange d'efforts et de loyauté de la part de ce dernier. La quatrième valeur est le fatalisme. Elle est fondée sur la présence du Boudhisme en Thaïlande. Enfin, la cinquième et dernière valeur utilisée est la croyance en la récompense future (delayed gratification). Cette valeur signifie que l'individu agit dans le présent pour les récompenses qu'il aura en contrepartie dans le futur.

Or ces valeurs ont une importance dans l'implication communautaire des individus et par conséquent sur leur capacité à développer une gestion environnementale. Les résultats de leur étude empirique montrent que l'implication communautaire dépend de manière cruciale des deux valeurs d'individualisme et de réciprocité des échanges retranscrites par la relation patron-client. Evidemment les individus revendiquant un certain individualisme s'impliquent moins dans la gestion communautaire. A l'inverse ceux qui rejettent la valeur de réciprocité patron-client s'impliquent plus dans la gestion communautaire. Cela indiquerait qu'ils sont moins traditionnels et conformistes et peuvent en conséquence plus facilement questionner les modes de comportement en vigueur. L'impact de cette valeur est souligné également par Buenavista (1998) dans le cas des Philippines. Il insiste sur le pouvoir que certaines valeurs confèrent à certaines personnes. La relation patron-client est une relation hiérarchique dont les principes de réciprocité sont la générosité d'un côté et la loyauté de l'autre. Cette relation peut être à l'origine de comportements dévastateurs pour l'environnement. Dans son étude sur les Philippines, l'auteur insiste sur le fait que les patrons les plus généreux avec leurs clients sont ceux qui continuent de pêcher à la dynamite, malgré l'interdiction légale de le faire. Par réciprocité, la loyauté des clients est acquise. Dans ce cadre, des communautés construites sur la valeur de réciprocité patron-client peuvent engendrer des modes de production insoutenables. Mais, surtout, ce qu'illustre cet exemple est le fait que certains individus peuvent manipuler ou utiliser les normes à des fins stratégiques, ici maintenir des relations commerciales et éviter la concurrence.

La gestion en commun des ressources naturelles n'est donc pas bonne en soi puisque certaines valeurs peuvent produire une faible implication et ne permettront pas de 
réduire les comportements opportunistes, tandis que d'autres valeurs supposent une implication forte mais peuvent faire l'objet de manipulation de la part de personnes peu soucieuses de l'environnement mais dont le pouvoir au sein de la communauté locale est suffisamment fort pour que les autres individus se plient aux règles qu'elles édictent.

Par ailleurs, ces résultats laissent de côté un autre aspect du problème : des populations ne participent pas aux décisions parce que précisément elles ne sont pas considérées comme pouvant localement être légitimes. Or l'exclusion de ces populations peut provoquer de sérieux effets néfastes sur la bonne gestion des ressources.

Deacon (1999) a montré à partir d'une étude sur plusieurs pays que de faibles rendements dans l'agriculture et un haut taux de déforestation étaient dus à une insécurité sur le bénéfice de la terre. Bohn et Deacon (2000) ont également montré que l'accroissement du risque de confiscation de la terre provoquait une déforestation plus forte. Des études sur le Kenya (Waiganjo et Ngugi 2001) ou sur la région himalayenne (Somanathan 1991) confirment de tels résultats. Or, les stratégies de pouvoir et d'accaparement de la ressource par certains individus de la communauté supposent également l'exclusion d'une autre partie de cette communauté. L'exclusion des bénéfices d'une ressource ou l'incertitude quant au bénéfice futur peut provoquer des dégâts majeurs sur celle-ci. Par exemple, une zone de forêt classée conduisant à des restrictions fortes par rapport à la situation avant son classement prive une partie de la population de certains usages. Si ces usages, par exemple le ramassage du bois pour le chauffage, la cuisine, etc., sont indispensables aux individus, les transgressions deviendront aisément la règle et la gestion en commun ne se montrera pas efficace. En revanche, en ayant pris appui sur la communauté, la politique de gestion aura renforcé les pouvoirs des autorités coutumières, ce qui peut en contrepartie accentuer les conflits avec les personnes contestataires transgressant les règles. En somme, la politique produira des tensions au sein de la communauté pouvant déboucher sur une division voire un éclatement. Une étude de cas sur la partie amazonienne du Brésil (Alston et al. 1999) appuie de tels phénomènes. Les conflits entre propriétaires de terres et squatters dégénèrent régulièrement sous forme violente.

43 Les effets pervers de l'exclusion d'une partie de la population sont potentiellement nombreux. Le principe du bouc-émissaire surgira d'autant plus facilement que les autorités coutumières auront $\mathrm{du}$ mal à maintenir leur pouvoir sur les membres irresponsables. Ainsi, les immigrants ou ceux n'étant pas de telle ou telle origine, ou arrivés plus récemment dans la zone se verront facilement accusés de tous les maux et pourront subir une répression. Les politiques de préservation de l'environnement peuvent pousser certains individus à modifier radicalement leurs relations avec les autres et des conflits peuvent apparaître pour l'accès à ces ressources; certains ayant l'impression d'être chassés ou exclus tandis que d'autres s'en trouvent les bénéficiaires et les gardiens. Le principe du bouc-émissaire devient dans un tel cadre une politique usuelle. Les maux subis par la communauté viennent de l'extérieur, en particulier des nouveaux arrivants. Ainsi, dans le cas de Madagascar, la responsabilité de la destruction des ressources est attribuée aux migrants. Les populations migrantes arrivées plus récemment sont montrées du doigt, elles sont stigmatisées comme ne possédant pas les mêmes valeurs (Samisoa 2001). De nombreux autres exemples vont dans le même sens. En Côte d'Ivoire, la dégradation des forêts classées est fortement attribuée aux populations migrantes qui ne respectent pas les règles coutumières (Koffi 2005). 
Tous ces exemples illustrent des partis-pris qui sont en fait loin de refléter la réalité. Les populations migrantes ne sont généralement pas plus responsables des destructions que les autres. Si les ressources naturelles se dégradent alors qu'elles font l'objet d'une gestion communautaire, c'est précisément en raison des fortes disparités de pouvoir au sein des communautés et des exclusions que cela provoque. Andriananja (2005) a bien montré sur le cas de la gestion forestière à Madagascar que les phénomènes de pouvoir et d'exclusion au sein des communautés produisaient des effets clairement néfastes sur la gestion des ressources. Les personnes exclues adoptent de fait des comportements de pillage, voire allument des feux de forêts en représailles de leur exclusion.

Ces exemples illustrent le fait que même si les règles sont collectivement décidées, certains acteurs peuvent être exclus du processus de décision. Or dans ce cas, non seulement la gestion en commun des ressources naturelles n'est pas automatiquement une solution efficace, mais elle peut aussi devenir une politique destructrice des relations entre les différentes populations sur un même territoire. Ainsi, le régime de propriété commune, même s'il établit des droits et obligations envers les différents membres, pose la question de l'exclusion des non-membres et de la réaction de ceux-ci face à leur exclusion. Or, dans ces exemples, il est clair que la propriété commune, faute de concerner réellement tout le monde, provoque des stratégies d'appropriation ou de dégradation.

La gestion en commun des ressources naturelles part du constat que les communautés locales peuvent être mieux à même que le marché ou le gouvernement de gérer les ressources naturelles. Ce constat se transforme vite en postulat impliquant une généralisation de ce mode de gestion, à tel point que, comme le souligne Méral (2003), une telle optique devient un véritable dogme et passe du statut d'outil pour une gestion plus efficace à celui d'objectif à atteindre. Une telle transformation de l'outil en objectif est problématique parce qu'elle ne se soucie plus de l'efficacité de l'outil. L'ambition de cet article était précisément de mettre en garde contre une telle dérive. Si l'outil peut s'avérer efficace, il ne l'est pas automatiquement.

Trois aspects critiques peuvent être rappelés ici.

Premièrement, la gestion participative au cœur de la démarche n'est pas une solution miracle. Elle s'articule avec des modes de gestion privée ou publique pour former des configurations institutionnelles originales. Chaque cas à traiter suppose donc un montage institutionnel qui lui est en partie spécifique, même si de nombreux recoupements peuvent être faits par catégories de cas à traiter. Cependant, la capacité de la gestion en commun à résoudre les conflits d'usage est liée à la mise en œuvre de règles et sanctions décidées collectivement. L'implication forte des populations par une réelle démocratie participative, qui vise avant tout à définir les règles de fonctionnement, prend tout son sens. Mais cet aspect interroge du même coup l'efficacité des montages institutionnels dont chaque configuration est originale en fonction des problèmes à traiter. Les populations locales ont-elles le même pouvoir de décision dans l'établissement des règles que les pouvoirs publics?

Deuxièmement, l'ancrage territorial des communautés et des institutions locales implique bien souvent une certaine hétérogénéité d'acteurs. La communauté ne peut être pensée comme une entité homogène. Les stratégies d'acteurs et particulièrement de pouvoir au sein des communautés ne peuvent être éludées. Si la question de l'impact du degré d'hétérogénéité sur l'efficacité de la gestion des ressources est loin de trouver une 
réponse ferme et définitive, elle ne peut être évacuée et les principaux risques concernant les ressources ainsi que les populations doivent mieux être cernés.

Troisièmement, ces risques découlent d'une part des stratégies d'acteurs qui peuvent s'appuyer sur les normes communautaires et les instrumentaliser au détriment des ressources naturelles, mais également des acteurs exclus du bénéfice de la ressource qui adoptent en représailles des comportements dévastateurs sur cette ressource.

Dans de telles conditions, il paraît pour le moins hasardeux de faire de cette perspective de gestion un modèle miracle applicable n'importe où et en toutes circonstances. L'utilisation de l'outil de la gestion communautaire sans précaution, devenant un objectif plutôt qu'un outil, nous amène à nous interroger sur la possibilité d'une certaine instrumentalisation des populations locales par des bailleurs de fonds "occidentaux" désireux de préserver l'environnement. Une gestion communautaire sans dialogue social sur les règles et leur légitimité au niveau local risque fort de s'avérer une préservation de l'environnement contre certaines franges de la population, dont les comportements en retour ont de grandes chances d'être dévastateurs sur cet environnement.

\section{BIBLIOGRAPHIE}

Acciaioli G. (2006), Environmentality Reconsidered: Indigenous To Lindu Conservation Strategies and the Reclaiming of the Commons in Central Sulawesi, Indonesia, Mimeo, University of Western Australia.

Agrawal A. (2005), "Environmentality: Community, Intimate Government and the making of Environmental Subjects in Kumaon, India”, Current Anthropology, 46 (2), pp. 161-190.

Agrawal A. (1994), "Rules, Rules Making and Rules Breaking: Examining the Fit Between Rule Systems and Resource Use", In E. Ostrom, R. Gardner et J. Walker (eds), Rules, Games and CommonPool Resources, Ann Arbor: University of Michigan Press.

Agrawal A. (1993), "Removing roles, attaching strings: Institutional arrangements to provide water", Indigenous Knowledge and Development Monitor, 1(3), http://www.nuffic.nl/ciran/ikdm/1-3/ articles/agrawal.html.

Alston L., Libecap G.D., Mueller B. (1999), Land Reform Policies, the Sources of Violent Conflict, and Implications for Deforestation in the Brazilian Amazon, mimeo, Department of economics, University of Illinois.

Andersen K.E. (1995), “Institutional Flaws of Collective Forest Management”, AMBIO, 24 (6), pp.349-353.

Andriananja H. (2005), « Institutions locales et gouvernance des ressources naturelles. Le cas de la gestion contractualisée de la station forestière de Manjakatompo ", In Ballet J., Radja K. [eds], Le capital social en action. Territoires et transferts, Paris, L'Harmattan, pp.113-130.

Ault D.E. et Rutman G.L. (1979), “The Development of Individual Rights to property in Tribal Africa", Journal of Law and Economics, 22, pp.163-182. 
Baland J-M. et Platteau J-P. (1999), “The ambiguous impact of inequality on local resource management”, World Development, 27 (5), pp.773-788.

Baland J.M. et Platteau J-P. (1996), Halting Degradation of Natural Resources; Is there a Role for Rural Communities?, FAO, Oxford University Press.

Ballet J. et Hamzetta B. (2003), « Le capital social comme protection sociale ? Le cas de la Mauritanie », Tiers Monde, XLIV, n¹75, 637-655.

Barrett C.B., Brandon K., Gibson C., Gjertsen H. (2001), "Conserving tropical biodiversity amid weak institutions", BioScience, 51, pp.497-502.

Bassett T.J. (1993), "Introduction : The Land Question and Agricultural Transformation in SubSaharan Africa", In Bassett T.J. et Crummey D.E. (eds), Land in African Agrarian Systems, Madison, University of Wisconsin Press.

Berkes F. (2006), The problematique of Community-Based Conservation in a Multi-Level Worl, mimeo, University of Manitoba, Canada.

Bohn H. et Deacon R. (2000), “Ownership Risk, Investment and Use of Natural Resources”, American Economic Review, 90, pp.526-549.

Buenavista G. (1998), Social Capital in Community-based Resource Management, Miméo Burt R.S. (1992), Structural Holes : The Social Structure of Competition, Cambridge Mass, Harvard University Press.

Ciriacy-Wantrup S.V. et Bishop R.C. (1975), “Common property As a Concept in Natural Resources Policy”, Natural Resources Journal, 15, pp.713-727.

Coleman J.S. (1988), "Social Capital in the Creation of Human Capital" , American Journal of Sociology, vol.94, supplément, S90-S120.

Daniere, A. et L. Takahashi (1997), "Environmental Policy in Thailand: Values, Attitudes and Behavior among the Slum Dwellers of Bangkok," Environment and Planning, C 15, 305-327

Daniere A. et L. Takahashi (1999), "Environmental Behavior in Bangkok, Thailand: A Portrait of Attitudes, Values and Behavior," Economic Development and Cultural Change, 47 (3), 525-558.

Daniere A. et L. Takahashi (2000), Social Capital and Environmental Management : Culture, Perceptions and Actions among Slum Dwellers in Bangkok, Mimeo.

Deacon R.T. (1999), "Deforestation and Ownership: Evidence from Historical Accounts and Contemporary Data", Land Economics, 75 (3), pp.341-359.

Demsetz H. (1967), “Toward a Theory of property Rights”, American Economic Review, 57 (2), pp.347-359.

De Rosny E. (1981), Les yeux de ma chèvre, Paris, Plon.

Fauroux E. (2003), « Structures micro-locales du pouvoir et gouvernance dans les villages de l'Ouest malgache », Cahiers du C3EDM, La gouvernance locale à Madagascar. Représentation, modélisation, participation, pp.2-18.

Froger G., Méral Ph., Herimandimby V. (2004), “The expansion of participatory gouvernance in the environmental policies of developing countries: the example of Madagascar", International Journal of Sustainable Development, 7 (2).

Hardin G. (1968), "The Tradegy of the Commons", Science, 162, 1243-1248. 
Isham J. (2000), "Can Investment in Social Capitl Improve Local Development and Environmental Outcomes? A Cost-Benefit Framework to Assess the Policy Options", mimeo, Middlebury College.

Jodha N.S. (1985), "Population Growth and the Decline of Common Property Resources in Rajasthan, India", Population and Development Review, 11 (2), pp.247-264.

Koffi K.J.M. (2005), Analyse économique de l'aménagement forestier dans une perspective de développement socialement durable en Côte d'Ivoire, Thèse pour le doctorat en Sciences Economiques, Université de Montpellier 1.

Mahieu F.R. (2001), Ethique économique, Paris, L'Harmattan.

McPeak J. (2005), "Individual and Collective Rationality in Pastoral production : Evidence from Northern Kenya”, Human Ecology, 33, pp.171-197.

Méral Ph. (2003) «L'évaluation économique des projets environnementaux dans le contexte de la gouvernance locale : quelles évaluations ? ", Cahiers du C3EDM, La gouvernance locale à Madagascar. Représentation, modélisation, participation, pp.104-120.

Méral Ph. (2004), Indicateurs économiques pour l'évaluation des transferts de gestion, Rapport, Service de Coopération et d'Action Culturelle, Ambassade de France, Madagascar.

Mors M. (1993), « Le principe de subsidiarité et la politique de l'environnement dans la communauté », Insee Méthodes, 39-40, 235-248.

Mukhopadhyay L. (2004), “Inequality, Differential Technology for Resource Extraction, and Voluntary Collective Action in Commons”, Ecological Economics, 49, pp.215-230.

Neupane H. (2003), "Contested Impact of Community Forestry on Equity: Some Evidence from Nepal", Journal of Forest and Livelihood, 2 (2), pp.55-61.

Nugent J.B. (1993), "Between State, Markets and Households : a Neoinstitutional Analysis of Local Organizations and Institutions", World Development, 21 (4), pp.623-632.

Ollagnon H. (1991), « Vers une gestion patrimoniale de la protection et de la qualité biologique des forêts ", Arbres, forêts et communautés rurales, Bulletin 3, 32-35.

Olson M. (1965), The Logic of Collective Action: Public Goods and the Theory of Groups, Cambridge, Harvard University Press.

Ostrom E. (1990), Governing the Commons, Cambridge, Cambridge University Press.

Ostrom E., Gardner R., Walker J. (1994), Rules, games and Common Pool Resources, University of Michigan Press.

Pelligra D. (1998), Errances bédouines, Paris, Editions de l'Aube.

Platteau J.-P. (1996), “The Evolutionary Theory of Land Rights as Applied to Sub-Saharan Africa: A Critical Assessment", Development and Change, 27, pp.29-86.

Platteau J-P. (2003), “Droits de propriété et gestion efficace des ressources naturelles”, Les séminaires de l'Iddri, 10, Conférence du 1 juillet, Paris.

Portes A. (1998), « Social Capital : Its Origins and Applications in Modern Sociology », Annual Review of Sociology, vol.24, pp.1-24.

Pretty J. et Ward H. (2001), "Social Capital and the Environment”, World Development, 29 (2), 209-227.

Ribot J.C. (1995), “From Exclusion to Participation: Turning Senegal's Forestry Policy Around?", World Development, 23 (9), pp.1587-1599. 
Samisoa (2001), « Migrations et déforestation sur le plateau calcaire de Belomotse-Vineta », in Sociétés paysannes, transitions agraires et dynamiques écologiques dans le Sud-Ouest de Madagascar, Razanaka S., Grouzis M., Milleville P. et Aubry C. (eds), CNRE-IRD.

Seabright P. (1993), "Managing Local Commons : Theoretical Issues in Incentive Design”, Journal of Economic Perspective, 7 (4), pp.113-134.

Somanathan E. (1991), "Deforestation, Property Rights and Incentives in Central Himalaya", Economic and Political Weekly, Jan 26, pp.37-46.

Wade R. (1988), Village Republics : Economic Conditions for Collective Action in South India, ICS Press Oakland.

Waiganjo C. et Ngugi P.E.N. (2001), The Effects of Existing Land Tenure Systems on Land Use in Kenya Today, mimeo, International Conference on Spatial Information for Sustainable Development, Nairobi, Kenya, 2-5 Oct.

Weber J. (2000), « Conservation, développement et coordination : peut-on gérer biologiquement le social », In Compagnon D. et Constantin F., Administrer l'environnement en Afrique. Gestion communautaire, conservation et développement, Paris, Karthala, pp.69-106.

\section{NOTES}

1. Je tiens à remercier particulièrement Philippe Méral, Mélanie Requier-Desjardins et Nicolas Sirven pour les discussions très éclairantes que j'ai eues avec eux sur ce sujet. Cet article est issu d'un texte beaucoup plus large, intitulé « Prendre en compte la dimension sociale des politiques environnementales : un enjeu essentiel » présenté au Séminaire Interdisciplinaire sur le Développement Durable (SIDD), Université des Sciences et Technologies de Lille-IFRESI, le 2 mars 2006. Je tiens à remercier très vivement l'ensemble des participants à ce séminaire pour leurs remarques très pertinentes dont certaines ont contribué à la rédaction de cet article. Je tiens également à remercier deux rapporteurs de la revue pour les précisions qu'ils m'ont obligé à apporter.

2. L'application contraignante des règles à l'ensemble de la communauté est une condition nécessaire dans un régime de propriété commune, sinon un tel mode de propriété ne saurait éviter l'opportunisme de certains.

3. Un trou structural est l'absence de liaison entre des contacts non redondants. Imaginons deux cas possibles. Dans le premier cas l'individu $\mathrm{E}$ a des contacts avec les individus A, B, et C, mais ceux-ci n'ont pas de contacts entre eux. Dans le second cas, E a toujours des relations avec A, B et C, mais A, B et C ont également des contacts entre eux. Il est évident que dans le premier cas $E$ possède un avantage en termes d'information sur $A, B$ et $C$, qu'il ne possède plus dans le second. Dans le premier cas il existe des trous structuraux, pas dans le second. Un trou structural donne donc un avantage à certains, ici $\mathrm{E}$, et un désavantage à d'autres, ici A, B et C. D'une manière générale, la structure des relations affecte l'accumulation du capital. 


\section{RÉSUMÉS}

La gestion en commun des ressources naturelles est devenue une problématique cruciale dans la gestion des ressources naturelles. Elle semble faire un nombre d'adeptes de plus en plus conséquent. Les vertus qui lui sont prêtées sont pourtant très discutables. L'objet de cet article est précisément d'en proposer une lecture critique. Il est montré que les fondements sur lesquels elle repose supposent une discussion plus approfondie sur ces conditions d'application. L'application sans précaution pourrait provoquer des effets dévastateurs aussi bien pour les ressources que pour les populations.

Community-based management of natural resources is now a main figure of natural resources management. It seems to have a growing number of adherents. Its virtues are meanwhile disputable. This paper proposes a critical insight. Grounds of this approach need a more serious discussion. The application without precaution could provoke negative effects for resources as well as for population.

\section{INDEX}

Mots-clés : capital social, gestion en commun

Keywords : social capital, Community-based management

\section{AUTEUR}

\section{JÉRÔME BALLET}

Jérôme Ballet est maître de conférence à l'université de Versailles Saint Quentin en Yvelines et chercheur au Centre d'économie et d'éthique pour l'environnement et le développement (UMR 063 C3ED (IRD-UVSQ). Il est éditeur de la revue Ethique économique/Ethics and Economics. 